\title{
An Efficient Multi-Expert Knowledge Capture Technique
}

\author{
Julius O. Okesola \\ Department of Computer and \\ Information Sciences \\ Tai Solarin University of Education \\ ljebu-Ode, Ogun State Nigeria
}

\author{
Oluwafemi S. Ogunseye \\ Department of Computer Science \\ University of Agriculture, Abeokuta, \\ Ogun State, Nigeria
}

\author{
Olusegun Folorunso \\ Department of Computer Science \\ University of Agriculture \\ Abeokuta, Ogun State Nigeria
}

\begin{abstract}
Capturing tacit knowledge from multiple experts in different location have not been exactly the easiest of activity for organizations and knowledge developers alike. In this research, the time tested and trusted Delphi technique is critically analyzed to allow for the automation of its processes. A simple prototype design is used to model the algorithm for the creation of an eDelphi; a framework prototype and a guide to system developers . The algorithm and the framework presented in this work will help in the creation of KMSs and web based systems that can capture multiple expert knowledge in a collaborative environment.
\end{abstract}

\section{General Terms}

Knowledge Management, Multiple Knowledge Capture, Delphi Technique, NLP, Semantic Analysis, Text mining, Machine Learning.

\section{Keywords}

Multiple Knowledge Capture, Knowledge Management, Delphi Technique, NLP

\section{INTRODUCTION}

Knowledge controls the world today. Be it academics, Industry, government or business, an organization is only as good as the knowledge of its workers. The realization of this is what led to the concept of knowledge management (KM) and knowledge management systems (KMS). Knowledge Management and its practices undergoes continuous academic research [1]. This is not strange considering the theories about the success KM can bring to an organization. [2] considers KM to be the capturing of Knowledge from past decision making for the application to current decision making with the express purpose of improving organizational performance. [3] defined knowledge as a "fluid mixture of experience, values, contextual information and expert insight that provides a framework for evaluating and incorporating new experiences and information". Although [4,5] and [6] were major proponents of the classification of knowledge as Tacit or Explicit, all the previous researchers agree that knowledge originates and is applied in the minds of people. It suffices to say that all knowledge started as tacit and eventually ends up as tacit. We imply a Knowledge ExternalizationInternalization cycle.

For organizations to get the much needed competitive advantage or achieve optimum performance, the tacit knowledge must be put to use in decision making and solving of problems. Many times this translates to multiple experts or Knowledge Sources (KS) externalizing their knowledge especially in complex problem domains when there is a dispersed knowledge about the subject matter [7]. The use of many KS helps because the interaction between the KS stimulates a synthesis of experience.
Especially in multinational organizations or international agencies, KSs might be dispersed, spread across different countries and regions of the world. Beyond the limits of technology and disparate technological advances between the developed and the developing countries of the world, beyond the limits of budget and time constraints we present an efficient method for capturing knowledge from multiple KSs based on the old, time-tested Delphi technique. The framework for such design is shown through our prototype called e-Delphi. This research is capable of influencing how KMS are designed improving on existing systems.

\section{THE DELPHI TECHNIQUE}

Delphi essentially implies a method for structuring a group communication process so that it is effective in allowing the group as a whole deal with complex problems [8]. Knowledge from multiple KS is gathered through an iterative survey process to find solution to crucial problems in a specific knowledge domain.

In describing the original Delphi technique which was entirely paper based, [9] explains that in the Delphi technique "Iterations refer to the feedback process. The process was viewed as a series of rounds; in each round every participant worked through a questionnaire which was returned to the researcher who collected, edited, and returned to every participant a statement of the position of the whole group and the participant's own position. A summarization of comments made each participant aware of the range of opinions and the reasons underlying those opinions".

Breaking this statements into an algorithm gives:

Step 1: A problem domain is identified and the problem defined.

Step 2: Experts are given ample explanation of the problem.

Step 3: Experts present their opinion on viable solutions to the problem

Step 4: Moderator accepts their solution and summarizes it.

Step 5: Moderator presents these summaries back to the each of the expert for further clarification.

Step 6: Steps 3, 4 \& 5 are repeated a few times and eventually a final summary is prepared when the opinions have converged into a consensus - the final solution.

\section{THE e-DELPHI METHOD}

There have been few attempts to partially or fully computerize the Delphi technique with varied levels of success. [10] lamented the scarcity and insufficiency of research or documented work in the area of automating the processes despite the proven value of the Delphi technique. In this section we present the methodology of the e-Delphi. The model also incorporates the desirable features of the Blackboarding technique [7]. 
The Delphi process can be continuously iterated indefinitely [11] but most of the original researches agree that three iterations is often sufficient to collect the needed knowledge and reach a consensus [12, 13, 9,14, and 15). The following algorithm is based on [9].

$$
\begin{aligned}
& 1.1 \text { The Algorithm } \\
& \text { 1. Problem this is defined by management. } \\
& \text { 2. TimeFrame } \longleftarrow \text { Specified by management } \\
& \text { 3. } \mathrm{N} \longleftarrow \text { Number of KSs to use } \\
& \text { 4. Repeat } \\
& \text { 5. fKnowledge } \longleftarrow \text { acceptKnowledge() } \\
& \text { 6. For each Expert ê } \in \text { E do } \\
& \text { 7. } \delta[\mathrm{i}] \longleftarrow \text { acceptKnowledge( }) / / \delta \text { is individual } \\
& \text { knowledge unit or opinion } \\
& \text { 8. For } \mathrm{i} \longleftarrow 1 \text { to } \mathrm{n} / / \text { where } n \text { is the number of } \\
& K S \text { that commented or shared their views } \\
& \text { 9. If ( } \delta[i]==\text { fKnowledge) then } \\
& \begin{array}{l}
\mu \longleftarrow \text { merge }(\delta \text {, fKnowledge }) ; \\
\text { output } \longleftarrow \operatorname{summarize}(\mu)
\end{array}
\end{aligned}
$$

10. Else

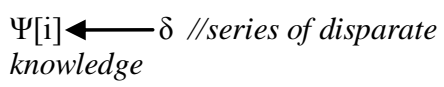$$
\operatorname{summarize}(\Psi[\mathrm{i}])
$$

$$
\begin{aligned}
& \text { 11. } \mathrm{T} \leftarrow \text { checkConvergence }(\Psi, \mu) \\
& \text { 12. Until } \mathrm{T} \text { is true or the number of iteration }=3 \text { then } \\
& \text { vote. } \\
& \text { 13. Show output } \\
& \text { 14. End. }
\end{aligned}
$$

\section{Explaining the algorithm in 8 steps:}

Step 1: the management presents a problem to the system and states a time frame for which the solution is needed.

Step 2: the experts receive alerts of this new problem and the explanation.

Step 3: the expert each propose a solution \{optional\} within the time frame

Step 4: their propositions are preprocessed and disambiguated to prepare it for summarization and comparison.

Step 5: the propositions are compared and the similar ones are merged or substituted for one another, then they are all summarized.

Step 6: if there are distinct view (i.e. no convergence) the result of the summary is presented to the KSs for further comments. The result of the majority position, the distinct view(s) are presented to each member and their own propositions (all summarized). This will give them a chance to review or further clarify their stance.

Step 7: the Step 6, is repeated until there are no distinct view or until the number of distinct views is below a particular value then they can be a vote for inclusion of the distinct view or exemption.

Step 8: the final report is presented to the KSs and the manager(s) as the consensus solution.

\section{THE e-DELPHI FRAMEWORK DESIGN}

For the sake of this framework and for the further clarification of the methodology this section show a sample (prototype) implementation, We used an Automatic Multiple and Single Document Summarizer and comparator module (See figure 1). This is the black box of the design. The internal workings of the (black box) system is discussed in this section.

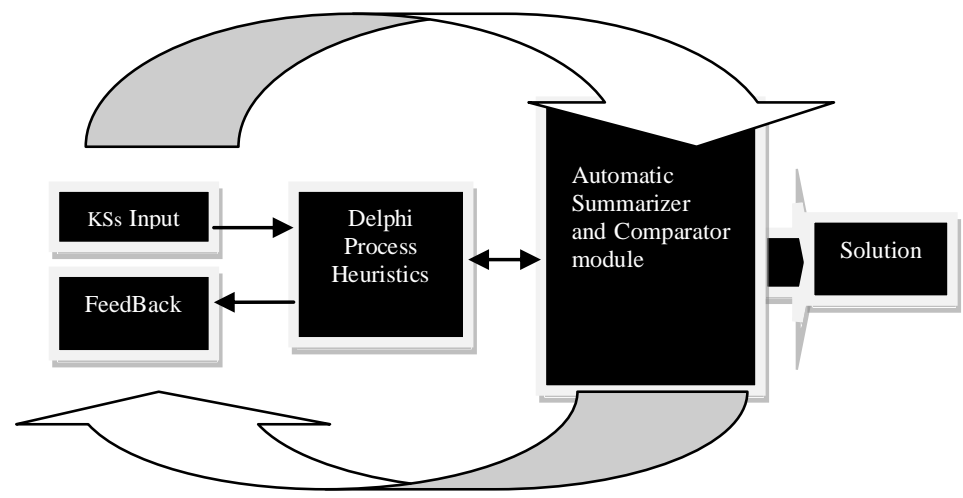

Figure 1: the Architecture of the e-Delphi

From the figure above (figure 1), it can be seen that each KS enter their opinion into the system. The system is controlled by the Delphi procedures \{the Delphi algorithm above\}regarded as heuristics. These heuristics control the interaction between the opinion of each expert and the "Black box". the expert get feedback from the system.

\subsection{Explaining the Architecture}

The prototype was designed with PHP 5.24 and Python and at the Backend is a MySQL database Server 5.0.45 running on an Ubuntu 9.04 Linux Server running Apache 2.2.4.

\subsubsection{Step 1: Input}

Each expert's input is converted to a random .txt file using a randomization algorithm.

\subsubsection{Step 2: Comparison}

The text in the text file is worked on by the summarization module described in the next subsection. The result of the statistical analysis is stored in our database which also contains words and synonyms (a thesaurus).

\subsubsection{Step 3: Summarization}

The text is merged based on their similarity and the number of expert with similar input is recorded also. The inputs are summarized, the merged files representing the group's position is also summarized by our auto-summarization tool described in subsection 4.3 and the result returned to the experts for further input, i.e. if the results have not converged to a single solution.

\subsubsection{Step 4: Voting}

Voting becomes necessary when there are views that are still not in agreement with the groups after three iterations.

The result is then returned to management as the proffered solution. 


\subsection{Semantic Analysis and Comparison Module}

KSs will submit knowledge with a few differences in the types of words used especially when they are all experts of the same problem domain. To ensure comparison is carried out their submission were converted to plain text and disambiguated using the semantic analysis and comparison module. The module breaks the terms into tokens. A list of synonyms to non pronouns and key terms is created from an inbuilt thesaurus, then other incoming documents are parsed and their key terms compared to the previous documents. The frequency of occurrence of key terms is noted the tone of the document is also considered. The text mining cum semantic analysis approach used is similar to the one used in [17] and Feldman et al[18].

\subsection{The Summarization Module}

after comparison and merger has taken place, each of the user input is sent to the summarization module. This module does its own comparison based on Natural Language Processing and creates summaries. It also takes note of similar documents which it confirms from the first module and then summarizes them as multiple documents into a single document and is presented as the group summary and stance. The Summarization tool that was used for this module DocSum was gotten from SourceForge.Net, A major source of Open Source Software. DocSum is used to create summaries for single and multiple documents using NLP based techniques (see figure 3 in appendix section). The software was redesigned to fit this application's purpose.

\subsection{Evaluating the Prototype}

We tested it using data from several past real and fictitious stockwatch magazines made by several reviewers about the same product. We used 5 real sources agreeing and 3 fictitious ones disagreeing as the initial input from experts and we set the threshold to 3 iterations. The system was able to allow us refine the opinions within 3 iterations an average of $96 \%$ of the time.

The accuracy of the summaries was sufficient for this prototype but not excellent. The system allowed for very quick processing .

Figure 2 shows the interface of the e-Delphi system.

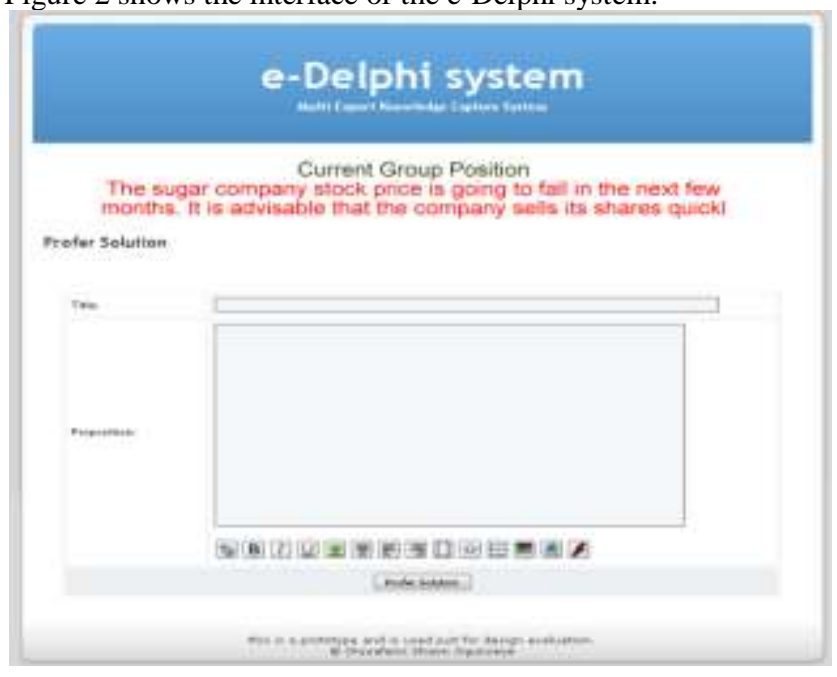

Figure 2: Screenshot of the e-Delphi System prototype

\section{DISCUSSIONS}

Some developers might feel the summarization and comparison tool duplicate effort and want to fuse them into one system. They are some commercial software and open source technology that can do both. Our implementation is by no means ideal, it's just a framework so any part can be redesigned or replaced. For other automatic document summarizing tools see [19]. We do not consider the variations in Delphi implementations.

\section{CONCLUSION}

[16] foresaw that an electronic Delphi would bear a lot of advantages. [10], stated in their work that an electronic Delphi opens the possibility for performance that will exceeds the composite performance curve. Problems with the Delphi technique have always been human factors and managerial problems [see 10,11]. In this work we have shown the steps that are needed to create and automate the processes of the Delphi technique. We even exemplified through the prototype e-Delphi system. This research will assist KMS developers in developing KMS systems that can actually help in true collaboration amongst experts to solve critical problems without having to be in the same place and will allow Experts contribute knowledge at anytime in an environment that fosters knowledge synthesis. The e-Delphi will find use in a broad spectrum of environment because of its versatility. Beneficiaries of this new technique will range from government planning, business and industry predictions, volunteer group decision, research etc.

\subsection{Future work}

Research should focus on how the e-Delphi model with whatever implementation technique that developers decide to use can be integrated into KMS and allied systems to allow for collaboration in problem solving. More work can also be done on improving our black box.

\section{ACKNOWLEDGMENTS}

Thanks to the Sourceforge Project and the Creator of the DocSum. 


\section{REFERENCES}

[1]. Zack M., McKeen J. and Singh S. 2009. Knowledge Management and Organizational Performance: an exploratory analysis. Journal of Knowledge Management, emerald group publishing Limited vol.13 no 6. 2009 pp 392-409.

[2]. Jennex Murray 2005. Case studies in Knowledge Management. Idea Group Publishing.

[3]. Davenport, T.H. and Prusak, L. 1998. Working knowledge: how organizations manage what they know, Harvard Business School Press, Boston, MA.

[4]. Polanyi M. 1958. Personal Knowledge: towards a post critical philosophy. Chicago: the University of Chicago Press.

[5]. Polanyi M. 1974. Knowing and Being. Chicago: the University of Chicago Press.

[6]. Nonaka I. 1994. A dynamic theory of organizational knowledge creation. Organization Science. Vol. 5, No. 1, pp 1437.

[7]. Awad E.M. \& Ghaziri H. 2004.Knowledge Management, Pearson Education, Inc ., Upper Saddle River, New Jersey.

[8]. Linstone H and Turoff M. 1975. The Delphi method: techniques and applications, Addison-Wesley

[9]. Ludwig, B. G. 1994. Internationalizing Extension: An exploration of the characteristics evident in a state university Extension system that achieves internationalization. Unpublished doctoral dissertation, The Ohio State University, Columbus.

[10]. Turoff M. \& Hiltz Starr R. 1996. Computer Based Delphi Process [online] http://web.njit.edu/ turoff/Papers/delphi3.html[accessed
[11]. Hsu C. \& Sandford Brian A. 2007. The Delphi Technique: Making Sense of Consensus, Practical Assessment, Research \& Evaluation. Vol 12, No 10.

[12]. Cyphert, F. R., \& Gant, W. L. (1971). The Delphi technique: A case study. Phi Delta Kappan, 52, 272-273.

[13]. Brooks, K. W. 1979. Delphi technique: Expanding applications. North Central Association Quarterly, 54 (3), 377385.

[14]. Ludwig, B. (1997). Predicting the future: Have you considered using the Delphi methodology? Journal of Extension, 35 (5), 1-4. Retrieved November 6, 2005 from http://www.joe.org/joe/1997october/tt2.html

[15]. Custer, R. L., Scarcella, J. A., \& Stewart, B. R. (1999). The modified Delphi technique: A rotational modification. Journal of Vocational and Technical Education, 15 (2), 1-10.

[16]. Witkin, B. R., \& Altschuld, J. W. (1995). Planning and conducting needs assessment: A practical guide. Thousand Oaks, CA: Sage Publications, Inc.

[17]. Ogunseye O.S., Folorunso O., Okesola J.O. \& Woodward J.R. 2010[in press], The EVAS Model: Solving EVoting Problems in Nigeria. Journal of Theoretical and Applied Information Technology.

[18]. Feldman R., Fresko M., Hirsh Haym, Aumann Y., Liphstat O., Schler Y. and Rajman M., 1998. Knowledge Management: A Text Mining Approach. Proceedings of the $2^{\text {nd }}$ Int. Conf. on Practical Aspects of Knowledge Management (PAKM98), Basel, Switzerland.

[19]. Das D. and Martins Andre F.T. 2007., a survey on automatic text summarization

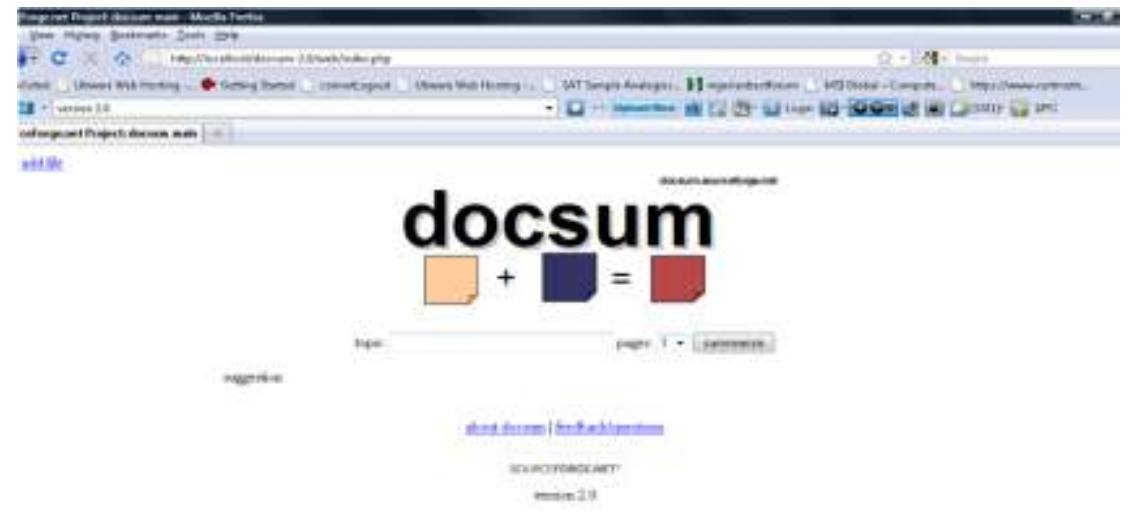

Figure 3: Screenshot of the DocSum, Automatic Document Summarizer 